\title{
BMJ Open Quality Better care: reducing length of stay and bed occupancy on an older adult psychiatric ward
}

Katherine Adlington, Juliette Brown, Laura Ralph, Alan Clarke, Tim Bhoyroo, Michael Henderson, Farai Boora, Marco Aurelio, Waleed Fawzi

To cite: Adlington K, Brown J, Ralph L, et al. Better care: reducing length of stay and bed occupancy on an older adult psychiatric ward. BMJ Open Quality 2018;7:e000149. doi:10.1136/ bmjoq-2017-000149

- Additional material is published online only. To view please visit the journal online (http://dx.doi.org/10.1136/ bmjoq-2017-000149).

$\mathrm{KA}$ and JB contributed equally.

Received 8 August 2017 Revised 19 September 2018 Accepted 7 October 2018

\section{Check for updates}

(c) Author(s) (or their employer(s)) 2018. Re-use permitted under CC BY-NC. No commercial re-use. See rights and permissions. Published by BMJ.

Mental Healthcare of Older Adults, East London NHS Foundation Trust, London, UK

Correspondence to Dr Juliette Brown; juliettebrown@nhs.net

\section{ABSTRACT}

Background Length of stay and bed occupancy are important indicators of quality of care. Admissions are longer on older adult psychiatric wards as a result of physical comorbidity and complex care needs. The recommended bed occupancy is $85 \%$; levels of $95 \%$ or higher are associated with violent incidents on inpatient wards.

Methods We aimed to reduce length of stay and bed occupancy on Leadenhall ward, a functional older adult psychiatric ward serving a population of just under 40000 older adults in two of the most deprived areas of the UK. At baseline in October 2015, the average length of stay was 47 days, and bed occupancy was at $77 \%$. We approached the problem using quality improvement methods, established a project team and proceeded to test a number of changes over time in line with the driver diagram we produced.

Results In 12 months, length of stay was reduced from an average 47 to an average 30 days and bed occupancy from $77 \%$ to $54 \%$. At the end of 2016 , the closure of some beds effected this calculation and we added an additional outcome measure of occupied bed days (OBD) better to assess the impact of the work. OBD data show a decrease over the course of the project from 251 to 194 bed days (a reduction of 23\%).

Conclusion The most effective interventions to address length of stay and bed occupancy on an older adult functional mental health ward were the daily management round and the high-level management focus on longerstay patients. The work depended on an effective community team and on the support of the quality improvement programme in the trust, which have led to sustained improvements.

\section{INTRODUCTION}

Length of stay and bed occupancy are important indicators of quality of care. Admissions are longer on older adult psychiatric wards as a result of physical comorbidity and complex care needs. The recommended bed occupancy is $85 \%$; levels of $95 \%$ or higher are associated with violent incidents on inpatient wards. ${ }^{1}$

Prior to this project, average length of stay for individuals admitted to Leadenhall ward was 47 days. There was a belief in the team that this figure was not indicative of clinical need, but was a result of a number of process factors which could be successfully addressed through quality improvement methods. Similarly, bed occupancy stood at baseline at 77\% and was considered high for the population served.

Leadenhall ward is a 19-bedded ward with an additional 7-bedded annex (total 26 beds) for adults over 65 with functional mental illness, part of the Tower Hamlets Centre for Mental Health at Mile End Hospital, under the management of Mental Healthcare of Older People Services in East London National Health Service (NHS) Foundation Trust. More than a third of admissions are people with schizophrenia or other psychotic illness; approximately 20\% have severe depressive illness and 20\% bipolar disorder, with predominantly manic presentation. Admissions also include people with acute stress reaction, personality disorder, mixed anxiety and depression, and delusional disorder. Some of our inpatients will have a comorbid cognitive impairment. The majority have comorbid physical illness, some severe and life limiting. Leadenhall Ward admits patients predominantly from the City of London, Hackney and Tower Hamlets, and on occasion, from other boroughs served by East London NHS Foundation Trust and by neighbouring trusts.

We aimed to eliminate delays in discharge and promote care in a less restrictive setting, that is, to discharge patients safely to their place of residence with ongoing care from community teams, with benefits to quality of care.

\section{BACKGROUND}

One in six of the UK population is currently aged 65 and over; by 2050, this is predicted to be one in four. ${ }^{2}$ In addition to organic mental illness, older adults can manifest any and all of the common, severe and enduring mental disorders. Some will have lived with recurrent 


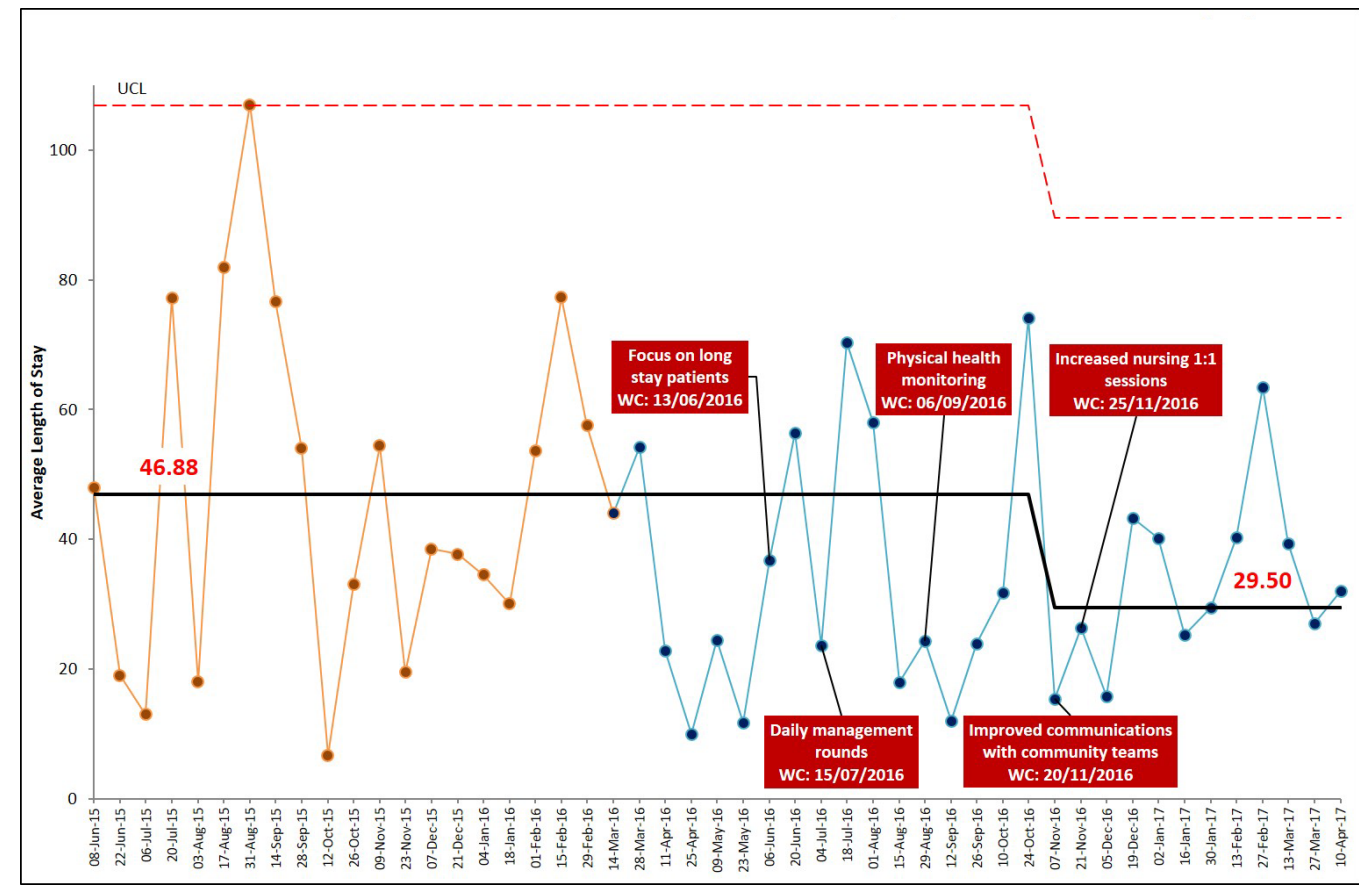

Figure 1 Average length of stay. WC, week commencing.

mental illness from a young age. Older age is commonly associated with depression, alcohol misuse and functional breakdown secondary to social isolation and a weakening of psychological resources. For every 1000 people over 65 , 250 will have a mental illness. ${ }^{3}$

In common with wider psychiatric services, most mental illness in older people is managed in the community. Inpatient care is highly specialised and is focused on caring for the most vulnerable, and those with greatest clinical need and complexity who cannot be managed in any other setting. ${ }^{45}$ Quality indicators specific to inpatient mental health services for older people can be used to inform and develop services and drive change. Length of stay is a quantitative indicator that can be used to measure quality of care on older adult inpatient wards and is affected by a number of different factors, such as severity of mental illness, compliance with treatment plan, physical health issues and discharge delays. Length of stay is highly variable across different settings, but prolonged inpatient admissions on older adult inpatient wards is a frequent occurrence and it is important to recognise the negative impact this has on patient care. Hospital length of stay

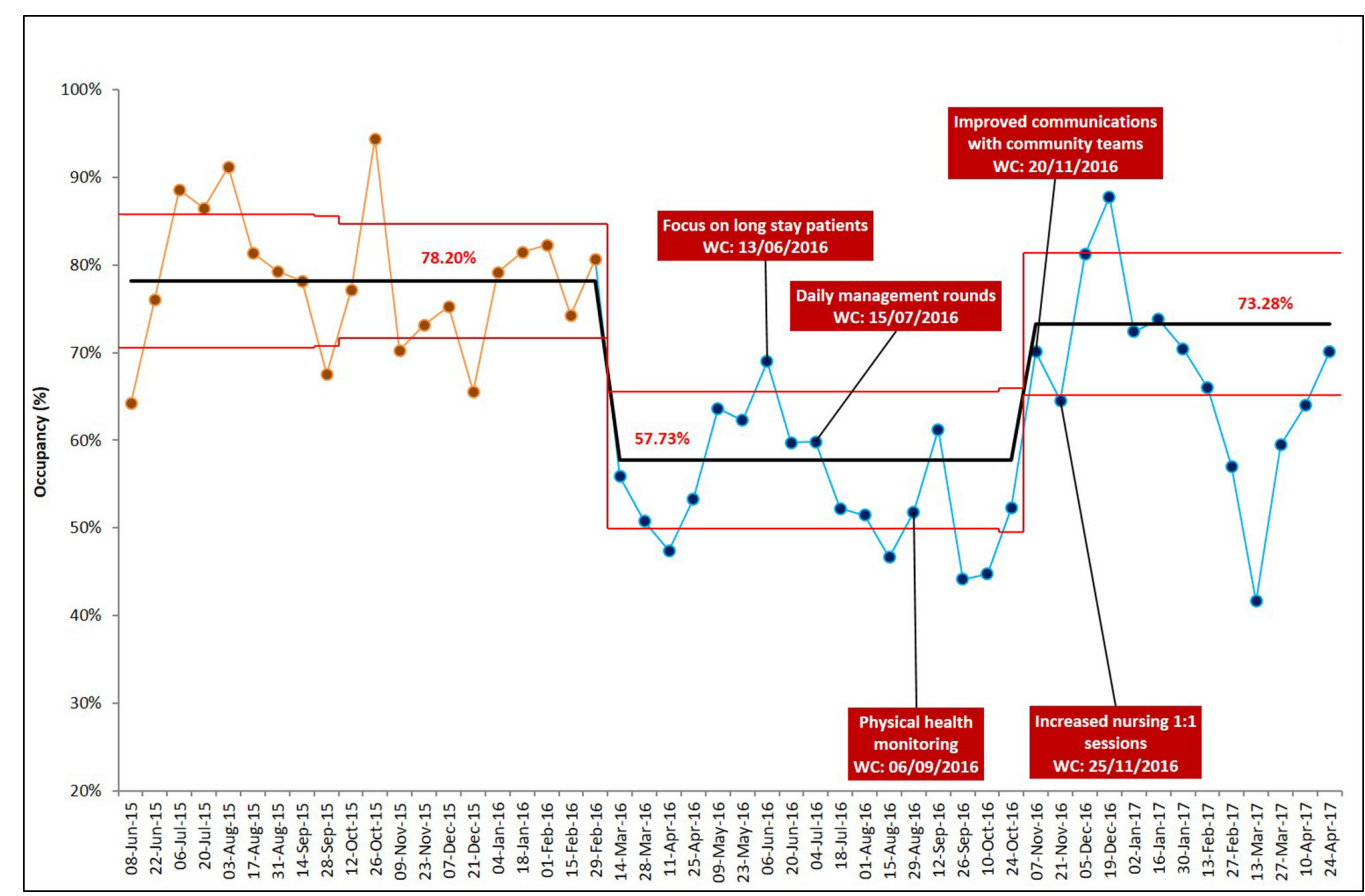

Figure 2 Bed occupancy. WC, week commencing. 
has been shown to predict functional decline for older adults, with a longer hospital length of stay associated with a greater likelihood of decline. ${ }^{6}$ In older patients with dementia, hospital admission is also associated with cognitive decline, increased rates of institutionalisation after discharge and higher mortality rates. ${ }^{78}$ Delayed discharge from inpatient services is a common cause of prolonged length of stays, with estimates of $16 \%-25 \%$ delayed discharges on older adult wards. ${ }^{9}{ }^{10}$ Reasons are thought to include delays in care home placement (approximately 10\% of admissions are discharged to a new address), limited availability or awareness of appropriate community services such as supported accommodation, rehabilitation services and intermediate care for older people. ${ }^{11}$

A number of policies have been proposed by different agencies and organisations to address increased length of stay on older adult wards. One of the key areas of focus relates to improving cross-agency working relationships, particularly between mental health services and social services.

Another key factor to consider when addressing prolonged length of stay is local service configuration. The reduction in overall bed numbers in recent years means that many services will now have 16-bedded to 20-bedded assessment wards providing care for patients from at least three or four locality sectors, each relating to corresponding Community Mental Health Teams. This has the potential for practical difficulties in traditional ward management (eg, multiple ward rounds, multiple different consultant styles of care across the ward, potential problems in terms of clinical leadership). The Royal College of Psychiatrists Faculty of Old Age Psychiatry have recommended in 2011 that in acute assessment areas, regular consultant ward reviews should be held at least twice weekly and additionally the consultant should be available to review urgent cases at any time during the working week. ${ }^{1}$ Adequate attention to both physical and mental health of older people can also impact length of stay. ${ }^{12} 13$

\section{METHODS: BASELINE MEASUREMENT}

A baseline was taken from June 2015 to March 2016 in which bed occupancy (bed occ) measured $77 \%$ and average length of stay (LoS) measured 47 days.

Occupied bed days (OBDs) was added as an outcome measure during the project as it is not affected by changes in numbers of available beds. The retrospective baseline measurement for this was 251 days.

In order to understand whether changes introduced as part of the project resulted in an improvement, a number of measures were adopted including

1. LoS (outcome measure). This is shown 4-weekly on a control chart for variable data (figure 1). LoS is calculated as discharge date minus admission date, at the point of patient discharge. The data are collected automatically from electronic systems and features on the trust quality dashboards.

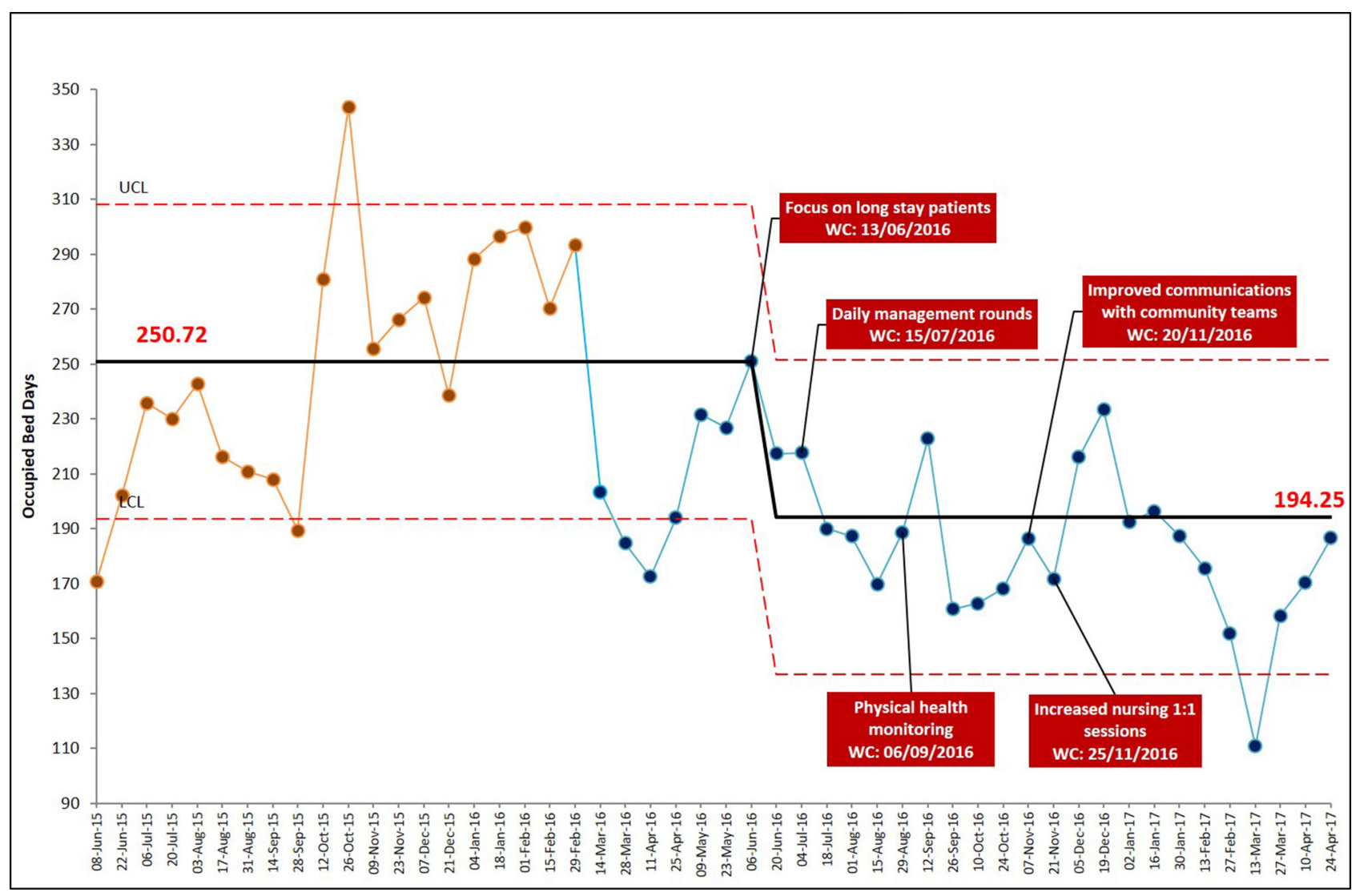

Figure 3 Occupied bed days. WC, week commencing. 
2. Bed occ (outcome measure). This is shown as a fortnightly percentage on a control chart for attribute data (figure 2). The numerator is taken as the number of occupied beds, excluding patients on leave, and the denominator is the total number of beds on the ward. The data are collected automatically from electronic systems and features on the trust quality dashboards. Bed occ is a ratio and is affected by number of available beds.

3. OBD (outcome measure) is shown 4-weekly on a control chart (figure 3). It was included as a measure during the project and retrospectively analysed, as a change in the total number of beds in October 2016 led to an increase in bed occupancy. OBD allows us to account for this change in bed numbers during the project. OBD as a measure is able to account for different bed numbers over time, as the denominator shifts according to available beds. OBD is the total number of available beds occupied expressed in days for the period calculated divided by the total number of available beds expressed in days for the period calculated. The data are collected automatically from electronic systems and features on the trust quality dashboard.

4. We also charted admission numbers per week and rates of readmission within 28 days (balancing measures). There was no increase in either over the course of the project (figure 4).

\section{METHODS: DESIGN}

The design of the project used the model for improvement, developed by Associates in Process Improvement, and the adopted method of the Institute for Healthcare Improvement.

The project lead is experienced in quality improvement (QI) methods, having received commendation for previous improvement work. Members of the team included a former improvement fellow/tutor of Improvement Science in Action with considerable experience in improvement work. We included all relevant parties. The membership of the project team included ward manager, consultant psychiatrist, sector higher trainee, junior doctors, occupational therapists (OTs) from the ward and community teams, social worker, service user representative and service director. We established a weekly meeting at the same time and day, and developed a driver diagram to form a visual display of relevant issues affecting the problem. We were supported from a QI Lead from the trust's central QI team, as well as a data and performance lead. They helped feedback progress and plan tests of change on a weekly basis. The data, in addition to the expertise of the team, were used to prompt change ideas.

\section{METHODS: STRATEGY}

The strategy for implementation was based on our driver diagram. We met as a team to develop an understanding

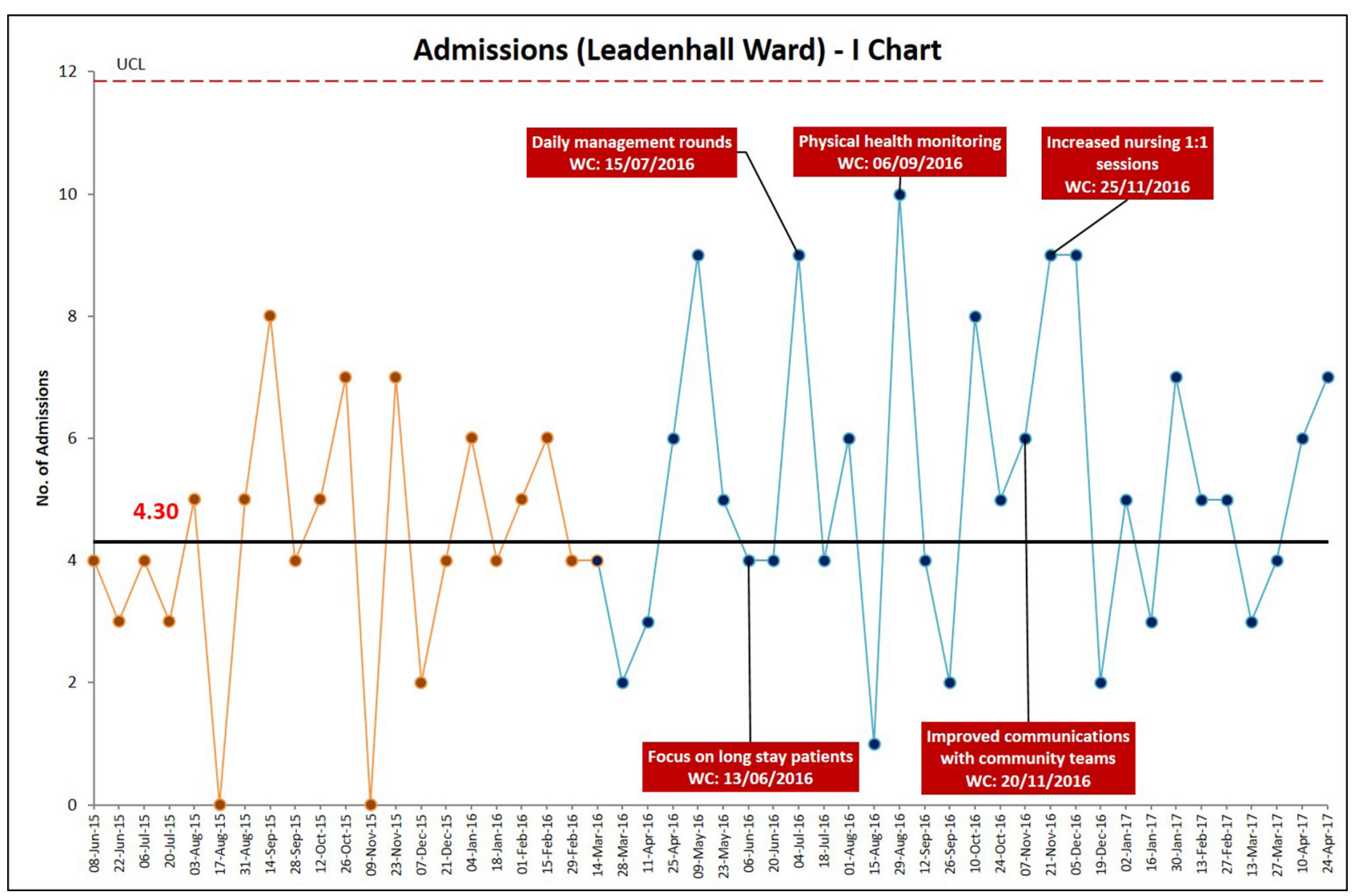

Figure 4 Admissions. WC, week commencing. 
of the primary drivers including admission process, bed management, discharge process and community services involvement. Of these four, we elected to focus on discharge process, bed management and community involvement, as these were considered the areas in which the team could exert the most impact.

Plan Do Study Act (PDSA) cycles were implemented using the weekly QI meetings. Each of the below drivers had separate PDSA cycles that were discussed weekly and implemented simultaneously. Where change ideas required more than one cycle, these were implemented sequentially.

\section{Discharge processes}

Under the driver of discharge process, we initiated a daily management round (huddle) of 1 hour in which all patients' primary needs were discussed by the MDT and tasks identified. We used PDSA to review and refine the management round process. This led to the use of a computer projection of the inpatient list and a table of relevant tasks to focus the discussion including completion of admission process, physical health review and initial OT assessment. Where the patient was likely to need assessment for a placement outside of their usual residence, this was flagged up at admission. Over the course of a number of PDSA cycles, the management round was refined. The impact of management rounds, attended by nursing, OT and medical staff, was a vastly improved process for decision-making at senior level on a daily basis. Patients were triaged for consultant, senior registrar $(\mathrm{SpR})$, junior doctor and nursing review and pressing issues delaying recovery were identified far more quickly. There was no overall reduction in psychiatric reviews.

The process was further refined through several PDSA. One intervention was that significant community staff for each patient were invited to the management rounds. Some of the other tasks included separate family meetings and reviews by care co-ordinators.

The result of the intervention was improved communication between the ward team (who worked different shifts) as notes from the daily round formed a document for daily review of progress on each patient. In addition, decision-making was far quicker as a result of the daily review of tasks.

As a second change idea, we focused attention on barriers to discharge of the five longest-stay patients. We planned to identify the five longest-stay patients at any given time in a rolling programme and for relevant staff to meet to look at issues in the discharge processes for each one. Studying this process, we found that the involvement of the service manager in close liaison with social services in the localities could help to overcome barriers to discharge. In the case of the longest-stay patients, these were predominantly social issues. Over the course of the project, this attention meant that patients who had been on the ward for many months were supported in their discharge and as a rolling programme the five longeststay patients were under review to identify what might be delaying discharge, outside of clinical issues. This intervention is seen as the successful implementation of a more integrated approach using the leverage of senior management to deliver better patient care.

A further change idea saw a dedicated social worker from one of the community teams asked to attend the management round on a weekly basis. The hypothesis behind this change idea was that attendance would improve communication of individual patient needs to social care colleagues in the localities. Unfortunately, due to staff sickness, the hypothesised benefit was not seen.

In a continuation of the project, we are considering another idea from our service user representative which is transitional discharge arrangements including proactive communication on a daily basis with patients on leave and following discharge to identify any issues that would lead to readmission/failed discharge. Our service user was able to convey the level of anxiety felt at the point of discharge in many cases. We feel that the attention paid to this area will improve quality of care in itself beyond any immediate impact on primary outcomes measures and may also help us to continue to achieve stable readmission rates.

\section{Bed management}

Under the driver of bed management, junior doctors suggested a process measure to ensure physical health monitoring was more regularly and proactively done, with the aim of reducing unexpected physical deterioration that might prolong inpatient stay and cause harm to patients. As a result of this monitoring, more patients had regular physical health monitoring. The proactive reviews identified in many cases physical health concerns requiring acute assessment and treatment. It was felt that the reviews did not contribute to a reduction in average LoS, and in some cases may have prolonged the inpatient stay, as patients were transferred to acute setting for management of physical health concerns. What was intended to improve average LoS contributed to better physical healthcare without an impact on length of stay. We found on studying the impact of this change idea that physical healthcare for our patients was more proactively reviewed.

We established the monitoring of regular, high quality 1:1 nursing support as a specific change idea. This idea stemmed from the involvement of a service user with recent experience of the ward environment, who felt that she-and other patients-did not always receive the expected 1:1 reviews on a daily basis from nursing staff. The view was that the lack of this input could significantly impact on recovery. As a result, the ward manager instigated a process to ensure that each patient received 1:1 reviews daily. The hypothesis behind the idea was that patients would benefit clinically and nursing staff would have a clearer sense of the mental state of their patients, which could be communicated at management rounds to the ward team as a whole. On studying the impact of the PDSA cycle, we found the reliability of regular reviews was difficult to achieve at times of bed pressures. This led to 
more proactive work on staffing levels during periods of bed pressure. Work on this process is ongoing.

\section{Community services involvement}

Under the driver of community services involvement, communication between the community teams and inpatient setting was identified as an area that required focus. We tested a number of change ideas with a view to better communication between community and inpatient teams. We conducted individual PDSA for each idea, including email updates to care co-ordinators. These were felt to be lacking in detail and did not contribute to improved communication. As a result of this learning, care co-ordinators were invited more often to ward reviews. This was felt to improve communication marginally. In addition, higher trainees from the community contributed 1 PA/ week to ward reviews, which was felt to improve the transition between ward and community, but did not make a significant impact on the main outcome measures of the project. Running a unit with intake from multiple localities presents significant communication challenges. We continue to work on better methods of communicating between the community teams and inpatient teams, and plan to use Skype for Business recently introduced by the trust to enable care co-ordinators to input without the need to travel long distances.

\section{RESULTS}

In the first year of the project between March 2016 and March 2017, LoS on average reduced by $36 \%$ from 47 days to 30 days. The shift in LoS began in October 2016, reflecting the impact of focus on longest stayers and on a daily management round. Bed occ was reduced from $77 \%$ to 55\%, with the major shift occurring in April 2016. OBDs were reduced at the same time. The data viewed in conjunction indicate that we did not simply reduce admission length only to admit an increased number of patients. Rather, we maintained the work in the community to avoid unnecessary admission. During the period of continuous monitoring, we found that the number of admissions overall did not change. We had an average of three admissions per week. As a result of reduced stays and a stable admission and re-admission rate, bed occupancy—-measured most robustly by OBDs—also reduced.

Results are represented in the following figures: 'figure 1 length of stay' (June 2015 to April 2017); 'figure 2 bed occupancy' (June 2015 to April 2017); 'figure 3 occupied bed days' (June 2015 to April 2017); 'figure 4 admissions' (June 2015 to April 2017).

An increase in the percentage of bed occupancy in November 2016 is due to the closure of a number of inpatient beds.

There were some change ideas that on analysis we felt yielded little impact. Others were much more successful. The most successful interventions were the addition of a daily management round leading to swifter decision-making. Dedicated work on the longest-stay inpatients was also very fruitful. The work has also relied on effective community teams who maintained stable admission rates. Other change ideas were felt to improve care without contributing to the specific aims of the project. We felt that this demonstrated the wider benefits of the increased attention to process generated by improvement work.

The gains of this project have been further sustained in the period following this analysis. Over the year April 2017 to March 2018, the average LoS has been 34 days. The continuation of the daily decision and task-focused management round has had a major impact on the sustainability of the gains. We have maintained our additional focus on longest-staying patients. Delays to discharge including social care issues are being proactively raised at senior management level. At ward level, improvements to communication between inpatient and community teams have also contributed to sustainability.

\section{LESSONS AND LIMITATIONS}

This project aimed at reducing LoS and bed occupancy on Leadenhall older adult psychiatric ward in Tower Hamlets. At baseline, the average LoS was 47 days, and bed occupancy at $77 \%$. In the first year of the project between March 2016 and March 2017, LoS on average reduced from 47 days to 30 days. These results exceeded our expectations, and the work on the project continues in order to sustain the gains.

Many changes implemented through the QI project were sustained on completion of the project. For example, the daily consultant management ward round continues to occur, as does the senior management review of longstay patients, liaison with social care leads and efforts to improve communication with community teams. We planned change ideas that would not require additional resource but rather a change in the structure or management process. This has meant that the LoS has remained at a low level of 34 days on average over the past year since this analysis, despite further operational changes including intake from five boroughs rather than three. A number of factors were thought to contribute to the project success and we benefited from

- The involvement of relevant professionals and a service user with recent experience of the ward.

- Using a method of improvement that allowed learning through continuous iterative tests of change.

- Sustaining a regular meeting, supported with highquality data and with a focus on change ideas.

- The support of effective community teams (representing a wider inter-related system).

- The involvement of professionals with experience in QI methods.

As a trust, we have seen the impact of QI work across services, and some of the confidence we have in using these methods to tackle problems stems from this wider success.

The most useful ideas included the establishment of a daily management round, which leads to high-quality 
decision-making on a daily basis and improved communication across the team. The dedicated focus on longerstay patients was also felt to be beneficial.

We feel that some of these ideas could be transferable to other settings, but also feel that the essence of this type of work is ideas generated locally that are of relevance to local teams. The most transferable elements are the wider factors including regular meetings, sustained focus on change ideas and the support of professionals with expertise and enthusiasm for QI methods.

\section{CONCLUSION}

As stated above, length of stay and bed occupancy are important indicators of quality of care, and are longer on older adult psychiatric wards as a result of physical comorbidity and care needs. We set out to reduce LoS and bed occupancy on Leadenhall, an older adult psychiatric ward in Tower Hamlets. We saw the LoS reduce from 47 days to 30 days-a 36\% reduction. We saw OBDs drop from 251 days to 194 days — at a time when admission numbers and readmission rates remained stable.

We felt the most effective interventions were the daily management round and the high-level management focus on longer-stay patients. The work depended on an effective community team and on the support of the QI programme in the trust. These ideas are likely transferable to other settings. Other elements that could be shared in other settings include the wider approach characteristic of improvement methods-including regular dedicated time, relevant staff engagement, service user involvement and a focus on learning through tests of change.

Acknowledgements Our service user representative who contributed and implemented change ideas. Kiwoong Park and Hau Lam Clara Fong for help in data collection. Dr Selim Kessab for physical health monitoring of patients on Leadenhall Ward. Michael Marin for coaching and help with data collection. Auzewell Chitewe for support in design of the project at the initial stages. All the staff on Leadenhall Ward, in the community mental health teams for older adults and the staff at East London NHS Foundation Trust for their contribution to this work.

Contributors KA contributed to the QI project, co-wrote and submitted the paper. JB helped coordinate the QI project, co-wrote and submitted the paper. LR, AC, $\mathrm{TB}, \mathrm{MH}$ and FB contributed to the QI project. MA contributed to the QI project and commented on the paper. WF oversaw the QI project and the writing of the paper.

Funding They received resources and support from the East London NHS Foundation Trust QI department.
Competing interests None declared.

Patient consent Not required.

Ethics approval This work met criteria for operational improvement activities exempt from ethics review.

Provenance and peer review Not commissioned; externally peer reviewed.

Open access This is an open access article distributed in accordance with the Creative Commons Attribution Non Commercial (CC BY-NC 4.0) license, which permits others to distribute, remix, adapt, build upon this work non-commercially, and license their derivative works on different terms, provided the original work is properly cited, appropriate credit is given, any changes made indicated, and the use is non-commercial. See: http://creativecommons.org/licenses/by-nc/4.0/.

\section{REFERENCES}

1. Royal College of Psychiatrists. Do the right thing: how to judge a good ward. Ten standards for adult in-patient mental healthcare. Occasional Paper OP79. 2011 http://www.rcpsych.ac.uk/pdf/OP79 forweb.pdf (accessed 6 Feb 2017).

2. Cracknell R. The Ageing Population. 'Key Issues for the New Parliament 2010-House of Commons Library Research' Government's Actuary Department. 2010 http://www.parliament. uk/documents/commons/lib/research/key_issues/Key-Issues-Theageing-population2007.pdf (last accessed 6 Feb 2017).

3. Royal College of Psychiatrists. Age discrimination in mental health services: making equality a reality. Royal College of Psychiatrists Position Statement PS2/2009. 2009 http://www.rcpsych.ac.uk/pdf/ PS02_2009x.pdf (last accessed 6 Feb 2017).

4. Pinner G, Hillam J, Branton T, et al. In-patient care for older people within mental health services. Faculty report FR/OA/1: Faculty of the Psychiatry of Old Age of the Royal College of Psychiatrists, 2011. (last accessed 6 Feb 2017).

5. Department of Health. Mental health policy implementation guide: adult acute inpatient care provision. london: Department of Health, 2002.

6. van Vliet M, Huisman M, Deeg DJH. Decreasing hospital length of stay: effects on daily functioning in older adults. J Am Geriatr Soc 2017;65:1214-21.

7. Sampson EL, Blanchard MR, Jones L, et al. Dementia in the acute hospital: prospective cohort study of prevalence and mortality. $\mathrm{Br} \mathrm{J}$ Psychiatry 2009;195:61-6.

8. Manning E, Timmons S, Barrett A, et al. The influence of dementia on one-year mortality following hospital admission, and place and cause of death. Irish J Med Sci 2014;183:319-20.

9. Naylor C, Bell A. Mental health and the productivity challengeimproving quality and value for money: The Kings Fund, 2010.

10. Lewis R, Glasby J. Delayed discharge from mental health hospitals: results of an English postal survey. Health Soc Care Community 2006;14:225-30.

11. Barker A, Bullock R. Delayed discharge in older people's mental health beds. Old Age Psychiatrist newsletter 2005. Autumn: 9.

12. Malyuk RE, Wong $C$, Buree $B$, et al. The interplay of infections, function and length of stay (LOS) in newly admitted geriatric psychiatry patients. Arch Gerontol Geriatr 2012;54:251-5.

13. Joint Commissioning Panel for Mental Health. Guidance for Commissioners of older people's mental health services. $2013 \mathrm{http}: / /$ www.jcpmh.info/wp-content/uploads/jcpmh-olderpeople-guide.pdf (last accessed 6 Feb 2017). 